Pure Appl. Chem., Vol. 85, No. 3, pp. 589-608, 2013.

http://dx.doi.org/10.1351/PAC-REP-11-11-12

(C) 2013 IUPAC, Publication date (Web): 11 January 2013

\title{
Fluorescence anisotropy measurements in solution: Methods and reference materials (IUPAC Technical Report)*
}

\author{
Marcel Ameloot ${ }^{1, \ddagger}$, Martin vandeVen ${ }^{1}, A$. Ulises Acuña ${ }^{2}$, and \\ Bernard Valeur ${ }^{3}$ \\ ${ }^{1}$ Universiteit Hasselt, BIOMED, Agoralaan, Building C, 3590-Diepenbeek, Belgium; \\ 2Instituto de Química-Física Rocasolano, CSIC, 119 Serrano, 28006-Madrid, \\ Spain; ${ }^{3}$ Département CASER, CNAM, 292 rue Saint-Martin, F-75141 Paris Cedex \\ 03, and Laboratoire PPSM, ENS-Cachan, 61 Avenue du Président Wilson, \\ F-94235 Cachan Cedex, France
}

\begin{abstract}
After recalling the basic relations relevant to both steady-state and time-resolved fluorescence polarization, it is shown how the values of steady-state polarized intensities recorded experimentally usually need to be corrected for systematic effects and errors, caused by instrumentation and sample properties. A list of selected reference values of steady-state fluorescence anisotropy and polarization is given. Attention is also paid to analysis of time-resolved fluorescence anisotropy data obtained by pulse fluorometry or phase and modulation fluorometry techniques. Recommendations for checking the accuracy of measurements are provided together with a list of selected time-resolved fluorescence anisotropy data as reported in the literature.
\end{abstract}

Keywords: anisotropy; fluorescence; IUPAC Analytical Chemistry Division; IUPAC Organic and Biomolecular Chemistry Division; IUPAC Physical and Biophysical Chemistry Division; photoluminescence; polarization; standardization.

\section{CONTENTS}

I. INTRODUCTION

II. DEFINITIONS AND BASIC RELATIONS

III. MEASUREMENT OF STEADY-STATE EMISSION ANISOTROPY

III.1 Differential sensitivity: $G$-factor

III.2 Geometry bias and "magic angle"

III.3 Blank subtraction

III.4 Other sources of systematic errors

III.4.1 Nonturbid samples

III.4.2 Turbid samples

III.4.3 Polarization bias due to sample holder and cuvette orientation, intensity reducers

III.4.4 High aperture excitation and detection

III.4.5 Detector geometry

III.4.6 Fiber optic bias

III.4.7 Checking stray light rejection

\footnotetext{
*Sponsoring bodies: IUPAC Physical and Biophysical Chemistry Division; IUPAC Organic and Biomolecular Chemistry Division; IUPAC Analytical Chemistry Division: see more details on p. 605.

¥Corresponding author: E-mail: marcel.ameloot@uhasselt.be
} 
III.5 Checking the accuracy of polarization measurements

III.6 Polarized excitation spectra

IV. TIME-RESOLVED FLUORESCENCE ANISOTROPY

IV.1 Expressions for time-resolved polarized intensities and fluorescence anisotropy

IV.2 Matching time-resolved polarized decays

IV.3 Data analysis

IV.3.1 Pulse fluorometry

IV.3.2 Phase and modulation fluorometry

IV.4 Checking the accuracy of time-resolved anisotropy measurements

IV.5 Identifiability analysis of time-dependent fluorescence anisotropy

MEMBERSHIP OF SPONSORING BODIES

ACKNOWLEDGMENTS

REFERENCES

\section{INTRODUCTION}

The measurement of stationary and time-resolved linearly (or plane) polarized fluorescence in solution is an important method of investigating physical and chemical molecular properties, as, for example, the electronic structure and solvent-solute interactions of small molecules, the conformation and dynamics of natural and synthetic macromolecules, etc. In addition, a growing number of very sensitive and specific analytical methods are based on the determination of changes in fluorescence polarization. The theoretical aspects underlying the emission of polarized fluorescence are presented with great detail in a number of general and specific textbooks [1-4] and journal publications. Conversely, the practical aspects of the corresponding experimental techniques, including the description of the most frequent sources of systematic errors that corrupt the measurement of polarized fluorescence intensity, are quite dispersed in the literature [4-19]. As a consequence, we would like to present here a comprehensive discussion of spectroscopic methods used currently in the measurement of fluorescence polarization, both steady-state and time-resolved, focusing on those practices that provide accurate and reproducible values of fluorescence polarization from liquid solutions. Methods that are specific for fluorescence microscopy and multiphoton applications will only be touched on briefly, as far as they have consequences for the availability and applicability of polarization standards.

\section{DEFINITIONS AND BASIC RELATIONS}

Fluorescence anisotropy $(r)$ is used to characterize the extent of linear polarization of fluorescence emission, resulting from photoselection from an optically isotropic sample. In the case of continuous excitation, the steady-state anisotropy $\bar{r}$ is given by $[3,20]$

$$
\bar{r}=\frac{I_{\|}-I_{\perp}}{I_{\|}+2 I_{\perp}}
$$

where $I_{\|}$and $I_{\perp}$ are the intensities ${ }^{*}$ measured with a linear polarizer for emission (the analyzer) parallel and perpendicular, respectively, to the electric vector of linearly polarized incident light (Fig. 1). The quantity $I_{\|}+2 I_{\perp}$ is the sum of the three mutually orthogonal emission components, and is in principle equal to the total fluorescence intensity $I$, which is polarization-independent.

\footnotetext{
*In the context of fluorescence anisotropy, only relative intensities are important. The term "intensity" should not be considered here as an absolute value. For the latter, terms such as "photon flux", "fluence rate", "irradiance", or "radiant power" (radiant flux) must be used. Additional information can be found on the IUPAC Gold Book website: http://goldbook.iupac.org/I03072.html.
} 


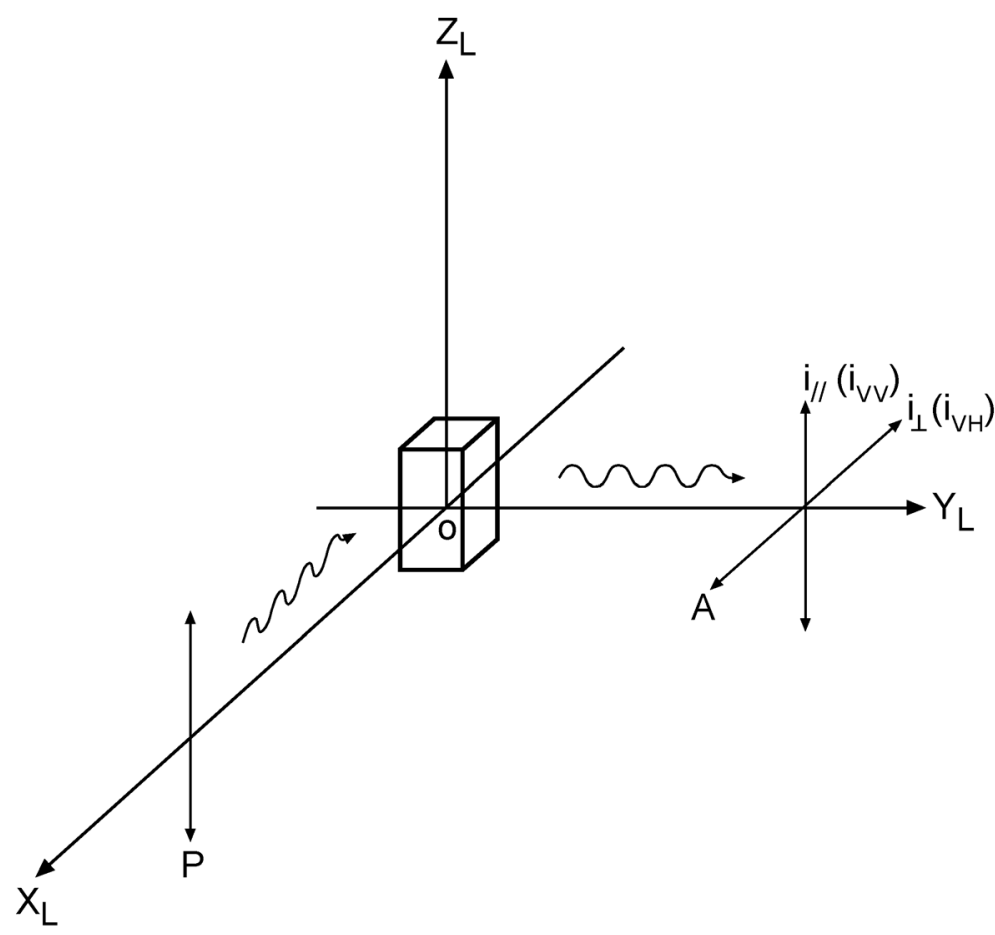

Fig. 1 The sample placed at the origin of the laboratory reference frame $\left(X_{\mathrm{L}}, Y_{\mathrm{L}}, Z_{\mathrm{L}}\right)$ is excited through a polarizer $\mathrm{P}$ with the principal axis oriented along the $Z_{\mathrm{L}}$ axis. The fluorescence emission is monitored through an analyzer A. The analyzer can be rotated so that the principal axis of the analyzer is either along the $Z_{\mathrm{L}}$ axis or the $X_{\mathrm{L}}$ axis. The corresponding intensities under ideal experimental conditions and under steady-state excitation are denoted as $I_{\|}$and $I_{\perp}$, or as $I_{\mathrm{VV}}$ and $I_{\mathrm{VH}}$. The time-resolved intensities under ideal experimental conditions and pulsed excitation are referred to as $i_{\|}(t)$ and $i_{\perp}(t)$, or $i_{\mathrm{VV}}(t)$ and $i_{\mathrm{VH}}(t)$; if the finite width of the excitation pulse has to be considered, the corresponding intensities are indicated as $I_{\|}(t)$ and $I_{\perp}(t)$, or $I_{\mathrm{VV}}(t)$ and $I_{\mathrm{VH}}(t)$.

In an alternative notation $I_{\|}$and $I_{\perp}$ are, respectively, represented as $I_{\mathrm{VV}}$ and $I_{\mathrm{VH}}$, where the first subscript corresponds to the orientation of the excitation polarizer and the second subscript to that of the emission polarizer. $\mathrm{V}$ and $\mathrm{H}$ stand for the vertical and horizontal direction, respectively, in a laboratory-based coordinate frame.

A closely related quantity is the fluorescence polarization ratio $(\bar{p})$, also called the degree of polarization [20]. For continuous illumination, the quantity $\bar{p}$ is given by

$$
\bar{p}=\frac{I_{\|}-I_{\perp}}{I_{\|}+I_{\perp}}
$$

The relation between $\bar{r}$ and $\bar{p}$ is given by

$$
\bar{r}=\frac{2 \bar{p}}{3-\bar{p}}
$$

However, in general, it is recommended that emission anisotropy rather than polarization be used because the former is normalized by the total emission intensity, namely, $I_{\|}+2 I_{\perp}$. 
Following an infinitely short pulse of polarized exciting light ( $\delta$-pulse) the time-resolved fluorescence anisotropy, or instantaneous anisotropy $r(t)$, is given by

$$
r(t)=\frac{i_{\|}(t)-i_{\perp}(t)}{i_{\|}(t)+2 i_{\perp}(t)}
$$

with $i_{\|}(t)+2 i_{\perp}(t)=i(t)$, the total time-resolved fluorescence intensity. From this equation, and the expression for $i(t)$ in terms of the polarized intensities, the time evolution of each polarized component of the fluorescence can be obtained

$$
\begin{aligned}
& i_{\|}(t)=\frac{i(t)}{3}[1+2 r(t)] \\
& i_{\perp}(t)=\frac{i(t)}{3}[1-r(t)]
\end{aligned}
$$

The relation between the instantaneous and steady-state anisotropy is given by

$$
\bar{r}=\frac{\int_{0}^{\infty} r(t) i(t) d t}{\int_{0}^{\infty} i(t) d t}
$$

The overall steady-state anisotropy of a mixture of $n$ components, where a component $i$ is characterized by an $\bar{r}_{i}$ value of anisotropy and contributing to the total fluorescence intensity with a fraction $f_{\mathrm{i}}=I_{\mathrm{i}} / I$, is given by

$$
\bar{r}=\sum_{i=1}^{n} f_{i} \bar{r}_{i}
$$

with $\sum_{i=1}^{n} f_{i}=1$.

Explicit expressions for the time-resolved anisotropy in the case of a mixture will be given below (see Section IV.4).

The term fundamental emission anisotropy $\left(r_{0}\right)$ pertains to a situation in which no depolarizing events occur subsequent to the initial formation of the emitting state, such as those caused by rotational diffusion or energy transfer. It is also assumed that only a single transition moment related to the absorption process is involved. In the most general case*, the (theoretical) value of the fundamental emission anisotropy just depends on the angle $\alpha$ between the absorption and emission transition moments in the molecular frame in the following way:

$$
r_{0}=\frac{1}{5}\left(3 \cos ^{2} \alpha-1\right)
$$

$r_{0}$ can take on values ranging from $2 / 5$ for $\alpha=0^{\circ}$ (parallel transition moments) to $-1 / 5$ for $\alpha=90^{\circ}$ (perpendicular transition moments). The corresponding values for $p_{0}$ are $1 / 2$ and $-1 / 3$. In spite of the assumptions mentioned above, the expression is frequently used to determine relative transition moment angles.

In the preceding paragraphs it has been tacitly assumed that one-photon excitation processes are considered. In general, multiphoton excitation has to be considered, which involves the absorption of

*Transition moments are not linear in very few cases of molecules having a particular symmetry, e.g., benzene, triphenylene, $\mathrm{C}_{60}$ [3]. 
two or more photons in the same quantum event. For such an excitation process with $l$ photons coming from the same source the initial emission anisotropy, $r_{0}^{(l)}$, is given by [9]

$$
r_{0}^{(l)}=\frac{2 l}{2 l+3}\left[\frac{3}{2} \cos ^{2} \alpha-\frac{1}{2}\right]
$$

where $\alpha$ refers to the angle between the dominant absorption and emission transition moments. For collinear transition moments $\left(\alpha=0^{\circ}\right)$ multiphoton excitation results in a more highly oriented excitedstate population as compared to one-photon absorption, which is reflected in larger values for $r_{0}$, for example, $r_{0}^{(2)}=\frac{4}{7}, r_{0}^{(3)}=\frac{2}{3}$.

In time-resolved fluorescence anisotropy, the theoretical value at time zero with $\delta$-pulse excitation is identified with $r_{0}^{(l)}$.

\section{MEASUREMENT OF STEADY-STATE EMISSION ANISOTROPY}

In a typical spectrofluorometer equipped with polarizers in the excitation and detection channels, the two polarized components of the fluorescence emission can be detected either simultaneously (T-format) or sequentially (L-format), usually in a direction at $90^{\circ}$ to the excitation beam. The T-format presents a series of advantages, for example, in collection time and when samples may photodegrade. However, balancing the electronic and optical properties of two detection channels may not be a simple task [8].

The values of the polarized intensities recorded experimentally usually need to be corrected for systematic effects and errors, caused by instrumentation and sample properties, as shown hereafter. The $\mathrm{T}$-format is the recommended method, provided that these instrumental issues are adequately taken into account.

\section{III.1 Differential sensitivity: G-factor}

The response of the optical elements and light detector placed in the emission channel can be very different for the vertical and horizontal components of the polarized fluorescence emission. The transmission of grating monochromators or pressure cell vessel walls is particularly sensitive to the linear polarization orientation of the incoming beam, an effect that can also be very strongly wavelength dependent [10]. This differential sensitivity of the detection channel can be corrected for (at each emission wavelength, $\lambda_{\text {em }}$ ) by the $G$-factor, which represents the ratio of the sensitivity of the detection system for vertically and horizontally polarized light:

$$
\bar{r}=\frac{I_{\mathrm{VVexp}}-G\left(\lambda_{\mathrm{em}}\right) I_{\mathrm{VHexp}}}{I_{\mathrm{VV} \exp }+2 G\left(\lambda_{\mathrm{em}}\right) I_{\mathrm{VH}} \exp }
$$

The value of $G\left(\lambda_{\mathrm{em}}\right)$ is best determined by exciting the fluorescent sample with horizontally polarized light [21], keeping unchanged the rest of the optical settings (slits, filters, etc.). In this way, the detection channel is illuminated by an identical amount of horizontally and vertically polarized light. If $I_{\mathrm{HV}}$ and $I_{\mathrm{HH}}$ are the actual experimental signals, then

$$
G\left(\lambda_{\mathrm{em}}\right)=\frac{I_{\mathrm{HV}}\left(\lambda_{\mathrm{em}}\right)}{I_{\mathrm{HH}}\left(\lambda_{\mathrm{em}}\right)}
$$

As indicated below, it may be necessary to subtract from each polarized component the background emission, as well. 
An alternative, but less general, way of suppressing the effect of the differential polarization sensitivity of the emission monochromator is by means of scrambler (depolarizer) plates. Scrambler plates are fused quartz wedges that rotate the incoming polarized beam by different amounts, depending on which part of the plate the light is traversing. As would be expected, the depolarizing effect of these plates is very sensitive to beam diameter and optical alignment. The larger the beam diameter impinging on the plate, the better the scrambling efficiency is. They are usually not $100 \%$ effective, and the $G$-factor should still be determined when using such a plate.

\section{III.2 Geometry bias and "magic angle"}

Total emitted fluorescence intensity is proportional to $I_{\|}+2 I_{\perp}$. A typical instrument in L- or T-format detects an intensity, which is not proportional to the total intensity unless the so-called magic angle settings are used. Several magic angle settings for the excitation and emission polarizer combination are possible $[4,10]$. The most commonly encountered optical configuration uses excitation light polarized in the vertical direction. In this case, the emission intensity must be recorded by orienting the polarizer in the emission path at the so-called magic angle of $54.7^{\circ}$ with respect to the vertical $\left(I_{54.7}\right)$. For instruments featuring linearly polarized laser illumination this is also the magic angle configuration of choice.

\section{III.3 Blank subtraction}

Parasitic emission from the solvent, cuvette, filters, etc. may be strongly polarized, hence greatly influencing the value of $I_{\mathrm{VV}}$, as in the case of leaking stray light consisting of scattered (Rayleigh) and Raman radiation. In contrast, the fluorescence emission from fast-rotating small-molecule impurities in fluid solution would be completely depolarized. If this detrimental emission cannot be eliminated, subtraction of the signal from an appropriate blank $\left(I_{\mathrm{b}}\right)$, namely, containing no exogenous dye, should be carried out for each polarized component:

$$
\begin{aligned}
& I_{\mathrm{VV}}=I_{\mathrm{VV} \exp }-I_{\mathrm{VV} \mathrm{b}} \\
& I_{\mathrm{VH}}=I_{\mathrm{VH} \exp }-I_{\mathrm{VH} \mathrm{b}}
\end{aligned}
$$

\section{III.4 Other sources of systematic errors}

\section{III.4.1 Nonturbid samples}

Ideally, samples should have no undissolved material nor dust particles present. Even with clear solutions, radiationless energy transfer and energy migration (fluorescence reabsorption, i.e., so-called inner-filter effects) are very effective in lowering the polarization of emission. A few measurements with increasing sample dilution usually suffice to detect these artifacts, although in some cases dilution may not help. For example, Buehler et al. [22] found the polarization of $15-\mu \mathrm{m}$ fluorescent latex spheres to be influenced by energy transfer, due to intraparticle interactions in the heavily stained beads.

\section{III.4.2 Turbid samples}

A more complex issue is the presence of Rayleigh or Mie scattering in turbid solutions, since it affects both the excitation and emission polarization. In the excitation channel, multiple scattering decreases very rapidly the polarization of the incident light, lowering the extent of photoselection [3]. Further depolarization may also occur in the emission channel. These effects have been discussed in detail $[23,24]$ and can be remedied by using cuvettes with a shorter optical pathlength [25].

\section{III.4.3 Polarization bias due to sample holder and cuvette orientation, intensity reducers}

Additional sources of depolarization may be inadvertent reflections in inner cuvette holder walls, which are not flat black, plastic cuvettes with walls consisting of partially aligned polymers that may act as polarizers, scratched and nonparallel cuvette windows, polarizer misalignment [26], too-wide slits [26], 
and solvent birefringence or optical activity. It should be noted, however, that polystyrene cuvettes have been successfully used for 1-anilino-8-naphthalenesulfonate (ANS) depolarization studies [10,25,27]. A pernicious systematic error can be introduced using $10 \times 3$ or $10 \times 2 \mathrm{~mm}$ cuvettes (internal pathlength dimensions). In this case, opening the excitation slit to decrease data collection time may result in an excitation beam that is too broad, hitting the inside wall (excitation beam propagation direction parallel to the longest pathlength) and possibly the solvent meniscus. Repeating an experiment but now by rotating the cuvette $90^{\circ}$ or using $\mu \mathrm{L}$ black quartz micro-cuvettes avoids this artifact. Thick-walled quartz pressure vessels for high-pressure protein denaturation studies may show similar effects. Pressure vessels present one of the challenges for polarization measurements, since the quartz windows have to be properly cut and a calibration run has to be performed to correct for the bulging windows, unless internal polarizers can be used [14,28-31]. Wavelength-independent stacked metal (e.g., copper) screens used to attenuate the emitted intensity may introduce multiple scatter from the round strands and distort the measurements. Polarization bias was also observed for accidentally tilted neutral density filters creating multiple non-overlapping illumination spots.

\section{III.4.4 High aperture excitation and detection}

Large collection angles of lenses or mirrors in the excitation and emission paths will depolarize the light focused on a sample and that emitted by a fluorophore [32]. When the collection solid angle is kept to within $\sim 15^{\circ}$ the error will be less than a few percent $[23,25]$. In some cases, it is possible to configure the emission polarizer to be the first element in the detection optical path, namely, before the collection lens, to avoid some of these effects.

\section{III.4.5 Detector geometry}

Glan-type polarizers with a wider opening allow for an increase in the detected light intensity. However, these polarizers tend to have a longer length, possibly leading to displacement of the passing beam upon rotation of the polarizer. When the beam is subsequently impinging on the round enclosure of an end-on photomultiplier, different parts of the detector cathode with varying response will be illuminated, resulting in a systematic polarization bias. This effect is even stronger when side-on photomultipliers are used.

\section{II.4.6. Fiber optic bias}

Multimode fiber optics may provide easy coupling via lenses and transport of light to sample and detector in some plate readers, stopped-flow and high-pressure instrumentation. Although total luminescence intensity may depend only slightly on fiber bending, the incoming polarization is at least partially scrambled [34], due to many internal reflections. To deliver well-polarized light to a sample, a polarizer has to be attached to the exit tip of the illumination fiber. On the emission side, the analyzing polarizer is attached to the entrance opening of the fiber optic [33]. When T-format emission is required, each of two emission fibers has to be fitted with its own polarizer and the relevant G-factor determination performed.

\section{III.4.7 Checking stray light rejection}

Scattered excitation light will give rise to an artificially high value for the detected $I_{\mathrm{VV}}$ component. The extent of the rejection of the scattered light by the emission filter or monochromator should be evaluated by using a purely scattering solution, recording the signal at the same detector gain, emission wavelength, and slit width as used in the actual experiment [25]. Ideally, the recorded intensity signal should not be very different from the dark count. Good scattering solutions can be obtained from freshly prepared diluted Ficoll (hydrophilic polysaccharide), colloidal silica (Ludox), or glycogen solutions. These should be checked regularly for any fluorescence impurity.

When using laser diode excitation light, spectral clean-up filters may be required to improve spectral purity. Superfluorescent background from the lasing medium and non-lasing spectral peaks emission (intrinsic to laser sources), masquerading as a sample fluorescence signal, may be present even $50 \mathrm{~nm}$ away from the lasing wavelength. The ill-conceived use of high diode laser power in combina- 
tion with a very low concentration of apparently surviving fluorophores can lead to an exceptionally high scattering environment. Even best available interference filters proved futile to reach mentioned dark count levels.

\section{III.5 Checking the accuracy of polarization measurements}

No precise polarization measurement can be carried out unless sample temperature is strictly controlled. This is an important consideration to avoid artifacts in both steady-state and subsequently time-resolved total fluorescence and anisotropy measurements [34]. Sample temperature equilibration by the use of stirrers in-between measurements eliminates any sample inhomogeneities. This may not be possible with some samples, as for example, fragile proteins, which will denature upon stirring, detected by coagulated protein on the bottom of the cuvette or foam on top of the solvent. This may seem a superfluous remark but since insertion of polarizers takes at least away $50 \%$ of each of the excitation and emission intensity, steady-state and time-resolved anisotropy measurements will take more time, leading to potential sample stability problems.

There is not yet a standardized method to test the accuracy of a fluorescence polarization measurement. A simple test consists of measuring the anisotropy of a diluted scattering solution [3,4], which should give ideally a value of 1 . A glycogen solution, a suspension of colloidal silica and Ficoll, with apparent absorbance $/ \mathrm{cm}<0.05 \mathrm{~cm}^{-1}$, are used for that purpose. The experimental values are usually slightly smaller, namely, because of the large apertures of the beams. Acceptable experimental values for narrow apertures are in the range of $\bar{r}=0.990-0.995$. Another convenient test is the measurement of the anisotropy of a fluorophore having a long excited-state lifetime (i.e., $\tau>15-20 \mathrm{~ns}$ ) in a fluid solution, so that fluorescence depolarization due to Brownian rotation is complete $(\bar{r}=0)$. A solution of ruthenium tris(bipyridyl), [Ru(bipy) $)_{3}$ [35], can be used for this test over a broad range of excitation wavelengths $(350-550 \mathrm{~nm})$. The use of 1-diethylamino-naphthalene-sulfonate (DENS) with an excitation maximum near $370 \mathrm{~nm}$ and a fluorescence lifetime of $30 \mathrm{~ns}$ [36] was also reported. A value also very close to zero has been reported [8] for an aqueous solution of fluorescein dianion excited at $348 \mathrm{~nm}$. Intermediate values of anisotropy can be achieved with compounds with excited-state lifetimes short enough so that randomization of orientations (due to Brownian rotation) following photoselection is not complete during the lifetime of the excited state. Phloxin B, rose bengal, and erythrosin B in aqueous solution buffered at neutral $\mathrm{pH}$ can be used for that purpose [37]. The experimental value of the fundamental anisotropy, also called limiting anisotropy, of perylene and xanthene derivatives in different solvents has also been reported [38].

The limiting anisotropy of rhodamine 101 was found to be equal to the theoretical value of $2 / 5$, so that its use as a standard for fluorescence polarization measurements was pointed out [39].

Table 1 lists a selection of reference values of anisotropy $\bar{r}$ and polarization $\bar{p}$. These values are given for information only since suitable standards should present a polarization value to a great extent independent of wavelength. The combination of various standards should cover the spectral region of interest with polarization values with the required sensitivity, accuracy, and repeatability. 
Table 1 Selected steady-state fluorescence anisotropy and polarization data as reported in the literature.

\begin{tabular}{|c|c|c|c|c|c|c|}
\hline Compound & Solvent & $T /{ }^{\circ} \mathrm{C}$ & $\lambda_{\mathrm{ex}} / \mathrm{nm}$ & $\lambda_{\mathrm{em}} / \mathrm{nm}$ & $\bar{r}(\bar{p})$ & Ref. \\
\hline PRODAN & Glycerol & 20 & $470-490$ & 520 & $\begin{array}{c}0.35 \\
(0.45)\end{array}$ & {$[40]$} \\
\hline Fluorescein & Glycerol-water 95:5 & 10 & $450-550$ & 516 & $\begin{array}{c}0.38 \\
(0.477)\end{array}$ & {$[6 \mathrm{~b}, 41]$} \\
\hline Rhodamine B & Glycerol-water $95: 5^{\mathrm{a}}$ & 10 & $540-580$ & 573 & $\begin{array}{c}0.39 \\
(0.492)\end{array}$ & {$[6 \mathrm{~b}, 41]$} \\
\hline \multirow[t]{2}{*}{ Rhodamine 101} & Glycerol & 23 & $500-600$ & 615 & $\begin{array}{c}0.38 \\
(0.479)\end{array}$ & [39] \\
\hline & PMMA thin film & 23 & $\begin{array}{l}500-585 \\
550-610\end{array}$ & $\begin{array}{l}615 \\
635\end{array}$ & $\begin{array}{c}0.40 \\
(0.50)\end{array}$ & \\
\hline \multirow[t]{2}{*}{ Erythrosin B } & Water & 20 & 514 & 570 & $\begin{array}{l}\sim 0.18 \\
(0.248)\end{array}$ & {$[42]$} \\
\hline & HEPES, pH 7.2, $50 \mathrm{mM}$ & 25 & 520 & 550 & $\begin{array}{c}0.243 \\
(0.325)\end{array}$ & {$[37]$} \\
\hline Phloxin B & HEPES, pH 7.2, $50 \mathrm{mM}$ & 25 & 520 & 560 & $\begin{array}{c}0.055 \\
(0.080)\end{array}$ & {$[37]$} \\
\hline Rose bengal & HEPES, pH 7.2, $50 \mathrm{mM}$ & 25 & 520 & 565 & $\begin{array}{c}0.251 \\
(0.335)\end{array}$ & {$[37]$} \\
\hline $\mathrm{Ru}(\text { bipy })_{3}$ & HEPES, pH 7.2, $50 \mathrm{mM}$ & 25 & 450 & 610 & $\begin{array}{c}0.00 \\
(0.000)\end{array}$ & {$[37]$} \\
\hline
\end{tabular}

a95:5 glycerol:water mixture (v:v), containing ca. $0.5 \mathrm{mM} \mathrm{NaOH}$.

\section{III.6 Polarized excitation spectra}

The excitation polarization spectrum represents the variation of the emission anisotropy registered at the emission wavelength $\lambda_{\mathrm{em}}$ as a function of the excitation wavelength $\lambda_{\mathrm{ex}}$. It is obtained experimentally by recording the variation of $I_{\mathrm{VV}}\left(\lambda_{\mathrm{ex}}\right)$ and $I_{\mathrm{VH}}\left(\lambda_{\mathrm{ex}}\right)$ and calculating the anisotropy as a function of $\lambda_{\mathrm{ex}}$, with $G\left(\lambda_{\mathrm{em}}\right)=I_{\mathrm{HV}}\left(\lambda_{\mathrm{em}}\right) / I_{\mathrm{HH}}\left(\lambda_{\mathrm{em}}\right)$. Since the emission wavelength is kept constant, a single determination of the $G$-value should suffice, unless large variations occur in the intensity of the exciting light that may change the geometry of the detecting optics. In this latter case, it is advisable to check the $G\left(\lambda_{\mathrm{em}}\right)$-factor for different values of $\lambda_{\mathrm{ex}}$.

\section{TIME-RESOLVED FLUORESCENCE ANISOTROPY [3,4]}

In a fluid environment, the initial uniaxial distribution of photoselected molecules is usually randomized by rotational diffusion, leading to depolarization of fluorescence. In the case of free rotation, the fluorescence anisotropy decays to zero, while for hindered rotation the anisotropy reaches a time-independent value at long times, $r_{\infty}$, called the residual anisotropy. The analysis of the temporal decay of the anisotropy yields direct information on the orientational dynamics, which depends on the size and shape of the rotating species and on the fluidity of its microenvironment. 


\section{IV.1 Expressions for time-resolved polarized intensities and fluorescence anisotropy}

For an excited species in a single type of isotropic environment, $r(t)$ is, in general, given by a linear combination of exponentially decaying functions

$$
r(t)=\sum_{j=1}^{m} \beta_{j} \exp \left(-t / \theta_{j}\right)
$$

where $\theta_{j}$ are the rotational correlation times. Obviously, the sum of the factors $\beta_{j}$ yields the fundamental emission anisotropy $r_{0}$.

The anisotropy decay of a general ellipsoid in an isotropic environment is given by a sum of five exponentials [3,4]. However, in practice no more than three exponentials can be observed because of ill-conditioning due to the non-orthogonality of exponentials in general [3].

The rotational correlation times depend on $D_{\perp}$ and $D_{\|}$, which are, respectively, the rotational diffusion constants for rotation around an axis perpendicular to the ellipsoid symmetry axis and around the ellipsoid symmetry axis. The rotational correlation times are functions of the shape and the elongation of the rotor, the viscosity of the solvent, and the temperature, but are independent of the excitation and emission wavelengths.

The time-resolved anisotropy of a mixture of $n$ emitting species may be obtained from the polarized intensity components in the following way. Assuming the total intensity decay $i(t)$ after $\delta$-pulse excitation can be written as

$$
i(t)=\sum_{k=1}^{n} \alpha_{k} \exp \left(-t / \tau_{k}\right)
$$

the polarized intensity components $i_{\|}(t)$ and $i_{\perp}(t)$ can generally be written as

$$
\begin{aligned}
& i_{\|}(t)=\frac{1}{3} \sum_{k=1}^{n} \alpha_{k} \exp \left(-t / \tau_{k}\right)\left[1+2 \sum_{j=1}^{m} L_{k j} \beta_{j} \exp \left(-t / \theta_{j}\right)\right] \\
& i_{\perp}(t)=\frac{1}{3} \sum_{k=1}^{n} \alpha_{k} \exp \left(-t / \tau_{k}\right)\left[1-\sum_{j=1}^{m} L_{k j} \beta_{j} \exp \left(-t / \theta_{j}\right)\right]
\end{aligned}
$$

where the factors $L_{k j}$ are equal to unity if the emission decay time $\tau_{k}$ is associated with the rotational correlation time $\theta_{j}$, and zero otherwise.

Two cases have to be considered. In the non-associative case, each of the decay times $\tau_{k}$ is associated with all the rotational correlation times $\theta_{j}$. In the associative case, each of $\tau_{k}$ corresponds to a particular set of $\theta_{j}[7,43]$.

Instead of a discrete set $\left\{\alpha_{k}, \tau_{k}, \beta_{j}, \theta_{j}\right\}$, one can also consider distributions of both fluorescence decay and rotational correlation times. For example, in the case where all the emitting species are assumed to display the same intrinsic anisotropy and rotational dynamics, the expressions are as follows:

$$
\begin{aligned}
& i_{\|}(t)=\frac{1}{3}\left[\int_{0}^{\infty} \alpha(\tau) \exp \left(-\frac{t}{\tau}\right) d \tau\left(1+2 \int_{0}^{\infty} \beta(\theta) \exp \left(-\frac{t}{\theta}\right) d \theta\right)\right] \\
& i_{\perp}(t)=\frac{1}{3}\left[\int_{0}^{\infty} \alpha(\tau) \exp \left(-\frac{t}{\tau}\right) d \tau\left(1-\int_{0}^{\infty} \beta(\theta) \exp \left(-\frac{t}{\theta}\right) d \theta\right)\right]
\end{aligned}
$$

where $\alpha(\tau)$ and $\beta(\theta)$ are, respectively, the distribution functions of the fluorescence decay times and the rotational correlation times. 
The intensities $i_{\|}(t)$ and $i_{\perp}(t)$ refer to very specific orientations of the excitation and emission polarizers. For the situation where the polarizing angles of the excitation and emission polarizer with respect to the normal to the excitation-emission plane are given by $\psi$ and $\xi$, respectively, the corresponding polarized fluorescence decay $i(\psi, \xi, t)$ can in general be expressed as

$$
i(\psi, \xi, t)=\frac{1}{3} \sum_{k=1}^{n} \alpha_{k} \exp \left(-t / \tau_{k}\right)\left[1+\left(3 \cos ^{2} \psi \cos ^{2} \xi-1\right) \sum_{j=1}^{m} L_{k j} \beta_{j} \exp \left(-t / \theta_{j}\right)\right]
$$

implying that $i_{\|}(t)=i\left(0^{\circ}, 0^{\circ}, t\right)$ and $i_{\perp}(t)=i\left(0^{\circ}, 90^{\circ}, t\right)$.

The expressions for the polarized intensities in the case of excited-state processes with speciesdependent Brownian rotational diffusion are more complicated and will not be discussed here. Explicit expressions can be found in ref. [44].

The anisotropy decay of a body subjected to rotational diffusion in a hindering potential cannot be expressed in closed form. In these cases, the anisotropy decay is described by an infinite number of exponentially decaying functions. An empirical expression consisting of a limited sum of exponentials and a constant term, the residual anisotropy, $r_{\infty}$, has often been used to approximate this decay [11,45-47]:

$$
r(t)=\sum_{j=1}^{m} \beta_{j} \exp \left(-t / \theta_{j}\right)+r_{\infty}
$$

\section{IV.2 Matching time-resolved polarized decays}

Obviously the same precautions as mentioned for the steady-state measurements can be repeated here. The $G$ factor can be calculated by measuring the photon counting rates for appropriate positions of the polarizers, as under steady-state conditions. An accurate method of matching the two decay curves $I_{\|}(t)_{\exp }$ and $I_{\perp}(t)_{\exp }$, that is, to correct for the differential sensitivity of the detection channel (see Section III.1), consists of comparing the polarized decay data with an accurately measured steady-state value of the emission anisotropy [48]:

$$
G=\frac{1-\bar{r}_{\exp }}{1+2 \bar{r}_{\exp }} \frac{\sum_{0}^{T} I_{\|}(t)_{\exp }}{\sum_{0}^{T} I_{\perp}(t)_{\exp }}
$$

with the subscript "exp" referring to experimentally collected data. The summation refers to the sum of the collected data for the selected analyzer orientation, and $T$ denotes a time for which the responses have decayed by about 2.5 to 3 orders of magnitude from their peaks [11].

The requirement for proper matching can be relaxed by adopting an appropriate analysis as is indicated below.

\section{IV.3 Data analysis}

Discrimination between different models, e.g., associative vs. non-associative, and parameter recovery can be most challenging even when the correct model is known. A proper data analysis protocol is therefore of utmost importance.

\section{IV.3.1 Pulse fluorometry}

It will be assumed that the data are properly collected and that artifacts are well taken into account, and that the collected data obey Poissonian statistics. The recommended method of analysis is by a nonlin- 
ear least-squares approach in which the correct weights of the data points are taken into account $[11,49]$. Various implementations of the actual least squares search are possible.

The polarized intensities $I_{\|}(t)$ and $I_{\perp}(t)$ are convolution products of the impulse response function of the photophysical system under study with the instrumental response function for each polarization, $g_{\|}(t)$ and $g_{\perp}(t)$ :

$$
\begin{aligned}
& I_{\|}(t)=\int_{0}^{t} g_{\|}(u) i_{\|}(t-u) d u=i_{\|}(t) \otimes g_{\|}(t) \\
& I_{\perp}(t)=\int_{0}^{t} g_{\perp}(u) i_{\perp}(t-u) d u=i_{\perp}(t) \otimes g_{\perp}(t)
\end{aligned}
$$

In an L-format type time-resolved fluorometer $g_{\|}(t)$ and $g_{\perp}(t)$ may not be substantially different, but this depends on the set-up conditions. If the decay times are much larger than the width of the excitation pulse, the emission anisotropy can simply be calculated from the experimental decays of the polarized components by

$$
r(t)=\frac{I_{\|}(t)-G I_{\perp}(t)}{I_{\|}(t)+2 G I_{\perp}(t)}
$$

Special attention has to be devoted here to perform the data analysis with the correct weights as combinations of measured data are considered [11].

Wahl [50] investigated the statistical accuracy of rotational correlation times, as determined by single-photon timing fluorometry. The relative errors in the correlation times are proportional to the inverse of the square root of the total count in the experiment and to the inverse of the fundamental anisotropy. As can be expected, these errors are functions of the ratio $\theta / \tau$ of the correlation time to the fluorescence decay time. The smallest errors are obtained when they have comparable magnitude. However, good estimates for the correlation times are obtained for a rather large interval of $\theta / \tau$ ratios [50].

Several approaches can be followed when the convolution effect has to be taken into account.

A. Analysis of combinations of the polarization intensity decays

A first method involves the construction of the so-called sum, $S(t)$, and difference, $D(t)$, curves:

$$
\begin{aligned}
& S(t)=I_{\|}(t)+2 G I_{\perp}(t) \\
& D(t)=I_{\|}(t)-G I_{\perp}(t)
\end{aligned}
$$

The sum curve is analyzed to yield the parameters $\left\{\alpha_{k}, \tau_{k}\right\}$ of the total fluorescence decay $i(t)$. The resulting parameters are then fixed in the subsequent analysis of the difference curve to obtain the parameters $\left\{\beta_{j}, \theta_{j}\right\}$ of the anisotropy decay $r(t)$.

This approach has some drawbacks. For a proper deconvolution of $S(t)$ and $D(t)$, the shapes of the instrumental response functions corresponding to $I_{\|}(t)$ and $I_{\perp}(t)$ have to be essentially identical, i.e., $g_{\|}(t)=g_{\perp}(t)$. Therefore, $I_{\|}(t), I_{\perp}(t)$, and the instrumental response function need to be measured in alternation. The correct weighting factors should be calculated for each data point [11].

B. Simultaneous analysis of the polarization intensity decays

In a second and more powerful approach, the collected polarized decays are not combined but used as such. The global analysis approach [51,52], in which different sets of related experimental data are analyzed simultaneously by linking parameters that are common to different fitting functions, has proved to be very efficient in the analysis of total fluorescence decays, and is also the method of choice for the analysis of fluorescence polarization data $[43,53,54]$. 
Simultaneous analysis of $I_{\|}(t)$ and $I_{\perp}(t)$ has a number of considerable advantages compared to the sequential analysis mentioned above:

- The excitation profiles of the two curves may be different, allowing for several simultaneous detection channels, as in a T-format configuration.

- Experiments performed at different timing calibrations to resolve multiple rotations occurring on different time scales can be easily incorporated in a single global analysis.

- The usual weighting factors for single-photon timing experiments can be used directly.

- $\quad$ All parameters of both $i(t)$ and $r(t)$ are simultaneously optimized in a single-step analysis.

- Instead of fitting for the correlation times $\left\{\theta_{j}\right\}$, one can also fit directly for the underlying diffusion coefficients $D_{\|}$and $D_{\perp}$ in a so-called target analysis [55].

- Experiments performed at various angles of the excitation and emission polarizer with respect to the normal to the excitation-emission plane can be simultaneously analyzed. This is especially important for studies on oriented samples.

- If desired, the pre-exponential factors $\left\{\alpha_{k}\right\}$ can be linked through the use of matching factors $G$. In principle, the matching factor $G$ does need to be known beforehand [53-56]. Obviously, using an experimentally determined value reduces the number of fitting parameters.

The global analysis approach has been shown to allow for a better parameter recovery at lower signal-to-noise ratios and has a significantly better model discrimination power [43]. As the statistical properties of the data are known, one can use these criteria to evaluate the fit of a model to the experimental data as described for total intensity decays (e.g., Boens et al. [57]).

Feinstein et al. tested the utility of an independently measured steady-state anisotropy value as a constraint in global data analysis [58]. The use of this constraint was found to significantly improve the precision and accuracy of recovered parameters particularly for viscosities at which the protein's rotational correlation time was much longer than the fluorescence lifetime.

The performance of the simultaneous analysis of unmatched polarized fluorescence decay curves collected under various experimental conditions, including reference convolution, has been investigated systematically for various model cases with zero residual anisotropy [56]. When the decay of the total fluorescence and the anisotropy are mono-exponential, the combination of decay traces collected at the two extreme orientations of the emission polarizer is sufficient for the parameter recovery when the rotational relaxation time is not too much different from the fluorescence relaxation time and is larger than the timing calibration of the channels. Different timing calibrations have to be utilized when the relaxation times in the anisotropy decay are widely different. The inclusion of polarized fluorescence decays collected at additional orientations of the emission polarizer can lead to improved accuracy and precision. In general, a more accurate parameter recovery and a better model discrimination are obtained when the relative weights of the exponential terms in the expression of the polarized intensity can be changed. Whenever feasible, the use of different excitation wavelengths is recommended. For high $\theta / \tau$ ratios, it is beneficial to vary the polarizer setting, even though the relative weight of the exponential terms in the emission anisotropy cannot be changed in this way. Fixing the initial anisotropy in the case of unmatched polarized decays has the same effect as fixing the matching factors to their proper values. Increasing the number of counts in the determination of $I_{\|}(t)_{\exp }$ and $I_{\perp}(t)_{\exp }$, i.e., collecting to a higher signal-to-noise ratio is more efficient than measuring at different settings of the emission polarizer.

It has been pointed out that for each decay trace the area under the observed and the adequately matched calculated decay curves must be the same [55,56]. Crutzen et al. [56] implemented this constraint in their analysis program to enhance the rate of convergence. 
C. Analysis of the experimental anisotropy

The experimental anisotropy $r_{\exp }(t)$ calculated by means of eq. 21 can be directly analyzed even if the decay times are not much larger than the width of the excitation pulse. In fact, the anisotropy calculated from the experimental data, $r_{\text {exp }}$, can be fitted directly according to eq. 24 [59]

$$
r_{\exp }(t)=\frac{g(t) \otimes[r(t) i(t)]}{g(t) \otimes i(t)}
$$

where $i(t)$ is the fluorescence intensity decay in response to $\delta$-excitation and $g(t)$ is the response function of the instrument, which is assumed to be independent of the orientation of the polarization, i.e., $g(t)=g_{\|}(t)=g_{\perp}(t)$.

When the anisotropy decay is much faster than the intensity decay, eq. 24 becomes eq. 25

$$
r_{\exp }(t)=\frac{g(t) \otimes r(t)}{g(t) \otimes 1}
$$

In the case of complex fluorescence decays, direct analysis by means of eq. 24 or 25 turns out to be an efficient method [60].

D. Case of distributions of decay and correlation times

Analysis of models involving distributions of both fluorescence decay and correlation times can also be implemented by the maximum entropy method (MEM) [61,62]. The $\alpha(\tau)$ profile is given from the initial analysis of the total fluorescence intensity $I(t)$ by MEM and is held constant in a subsequent analysis of $I_{\|}(t)$ and $I_{\perp}(t)$, which provides the distribution $\beta(\theta)$ of correlation times. Typically, 100 lifetime values equally spaced on the logarithmic scale within an appropriate interval are used for the analysis of $\alpha(\tau)$. The analysis of $\beta(\theta)$ can also be performed by starting with 100 rotational correlation times.

\section{IV.3.2 Phase and modulation fluorometry [3,4,63]}

Instead of recording separately the decays of the two polarized components, one measures the differential polarized phase angle $\Delta(\omega)=\phi_{\|}-\phi_{\perp}$ between these two components and the polarized modulation ratio $\Lambda(\omega)=m_{\|} / m_{\perp}$. Determination of the instrument response function is not required, nor is deconvolution. It is convenient to define the frequency-dependent anisotropy as follows:

$$
r(\omega)=\frac{\Lambda(\omega)-1}{\Lambda(\omega)+2}
$$

At low frequency, $r(\omega)$ tends toward the steady-state anisotropy, and at high frequency, $r(\omega)$ approaches $r_{0}$, the fundamental emission anisotropy (i.e., in the absence of rotational motions).

The following relations are used for data analysis:

$$
\begin{aligned}
& \Delta(\omega)=\tan ^{-1}\left(\frac{Q_{\|} P_{\perp}-P_{\|} Q_{\perp}}{P_{\|} P_{\perp}+Q_{\|} Q_{\perp}}\right) \\
& \Lambda(\omega)=\left(\frac{P_{\|}^{2}+Q_{\|}^{2}}{P_{\perp}^{2}+Q_{\perp}^{2}}\right)^{1 / 2}
\end{aligned}
$$

where $P_{\|}$and $Q_{\|}$are, respectively, the sine and cosine transforms of $I_{\|}(t)$, and $P_{\perp}$ and $Q_{\perp}$ are, respectively, the sine and cosine transforms of $I_{\perp}(t)$

$$
P_{\|}(\omega)=\int_{0}^{\infty} I_{\|}(t) \sin \omega t d t
$$




$$
Q_{\perp}(\omega)=\int_{0}^{\infty} I_{\perp}(t) \cos \omega t d t
$$

Phase data and modulation data can be analyzed separately or simultaneously (i.e., globally). In the latter case, the reduced chi-squared, which is to be minimized in the parameter search, is given by

$$
\chi_{\mathrm{r}}^{2}=\frac{1}{v}\left\{\sum_{i=1}^{N}\left[\frac{\Delta\left(\omega_{i}\right)_{\mathrm{exp}}-\Delta\left(\omega_{i}\right)}{\sigma_{\Delta}\left(\omega_{i}\right)}\right]^{2}+\sum_{i=1}^{N}\left[\frac{\Lambda\left(\omega_{i}\right)_{\exp }-\Lambda\left(\omega_{i}\right)}{\sigma_{\Lambda}\left(\omega_{i}\right)}\right]^{2}\right\}
$$

where $N$ is the total number of frequencies [63]. In this case, the number of data points is twice the number of frequencies, so that the number of degrees of freedom is $v=2 N-p$, with $p$ the number of freely adjustable fitting parameters.

In phase-modulation fluorometry, the use of $\chi_{r}^{2}$ deserves further consideration [34]. Values of the standard uncertainties (commonly referred to as standard deviations) $\sigma_{\Delta}$ and $\sigma_{\Lambda}$, must be assigned, and these values are in principle frequency-dependent. The determination of $\sigma_{\Delta}$ and $\sigma_{\Lambda}$ appears not to be obvious in practice. The important consequence is that the value of $\chi_{r}^{2}$ is not sufficient as criterion of a good fit. If the standard deviations $\sigma_{\Delta}$ and $\sigma_{\Lambda}$ are overestimated, the value of $\chi_{r}^{2}$ may be close to 1 , and an erroneous conclusion on the goodness of the fit may be drawn. This might be a common pitfall in phase-modulation fluorometry but the same would be true in time-domain measurements when such an overestimation of the standard deviations would take place. It should be emphasized that in this case only relative values can be used in accepting or rejecting a theoretical model.

\section{IV.4 Checking the accuracy of time-resolved anisotropy measurements}

Specific standards for calibration of instruments used to obtain time-resolved fluorescence anisotropy data are not presently available. The temporal resolution of time- and frequency-domain instrumentation can be tested by means of fluorescence lifetime standards, as detailed elsewhere [57]. In addition, the alignment of the excitation and emission polarizers can be checked with a very dilute scattering solution, as in a steady-state instrument.

Some literature data are listed in Table 2. The fluorescence is produced by one-photon excitation unless indicated otherwise. These are provided for information only and should not be considered as standard values stricto sensu. Dutt et al. [64] measured cresyl violet $\left(\lambda_{\mathrm{ex}}: 578 \mathrm{~nm}, \lambda_{\mathrm{em}}: 660 \mathrm{~nm}\right)$, nile blue $\left(\lambda_{\mathrm{ex}}: 578 \mathrm{~nm}, \lambda_{\mathrm{em}}: 690 \mathrm{~nm}\right)$, oxazine $720\left(\lambda_{\mathrm{ex}}: 620 \mathrm{~nm}, \lambda_{\mathrm{em}}: 720 \mathrm{~nm}\right)$, nile red $\left(\lambda_{\mathrm{ex}}: 578 \mathrm{~nm}, \lambda_{\mathrm{em}}\right.$ : $640 \mathrm{~nm}$ ) in various solvents, and extensive tables are reported.

Perylene in viscous solvents has become almost a standard in demonstrating the power of global analysis $[43,65]$. The sample is excited at two different wavelengths corresponding to absorption bands with nearly orthogonal polarizations. In a global analysis approach, the rotational correlation time can be linked over the two excitation wavelengths at a given temperature. Also, experiments performed at various temperatures allow linking but now over the pre-exponential factors of the time-dependent anisotropy. Depending on the imposed model, additional linking can be allowed for. The results obtained by Barkley et al. [66] have been confirmed by Balter et al. [67]. A quantum-mechanical study of the anisotropic behavior of perylene has been reported [68].

Finally, it should be noted that rhodamine 101 is of great interest as a fluorescence polarization standard because the value of $r_{0}$ measured both in rigid and in fluid media is equal to the theoretical one-photon fundamental emission anisotropy value of $2 / 5$ [39]. 
Table 2 Selected time-resolved fluorescence anisotropy data as reported in the literature.

\begin{tabular}{|c|c|c|c|c|c|c|c|c|}
\hline Compound & Solvent & $T /{ }^{\circ} \mathrm{C}$ & $\lambda_{\mathrm{ex}} / \mathrm{nm}$ & $\lambda_{\mathrm{em}} / \mathrm{nm}$ & $\tau / \mathrm{ns}$ & $r_{0}$ & $\theta / \mathrm{ns}$ & Ref \\
\hline Eosin-Y & Water & 20 & 514.5 & $>590$ & 1.069 & 0.396 & 0.157 & [54] \\
\hline \multirow[t]{5}{*}{ Resorufine } & 1-Propanol & 20 & 570 & 599 & $4.07 \pm 0.01$ & $0.36 \pm 0.02$ & $0.44 \pm 0.02$ & {$[56]$} \\
\hline & 1-Butanol & $27.0 \pm 0.1$ & 570 & 630 & n.r. & $0.37 \pm 0.01$ & $0.467 \pm 0.018$ & [73] \\
\hline & $\begin{array}{l}\text { 2-Methyl- } \\
\text { 1-propanol }\end{array}$ & $27.0 \pm 0.1$ & 570 & 630 & n.r. & $0.35 \pm 0.02$ & $0.599 \pm 0.021$ & [73] \\
\hline & 2-Butanol & $27.0 \pm 0.1$ & 570 & 630 & n.r. & $0.35 \pm 0.01$ & $0.523 \pm 0.024$ & {$[73]$} \\
\hline & $\begin{array}{l}\text { 2-Methyl- } \\
\text { 2-propanol }\end{array}$ & $27.0 \pm 0.1$ & 570 & 630 & n.r. & $0.34 \pm 0.03$ & $0.893 \pm 0.069$ & [73] \\
\hline \multirow[t]{4}{*}{ Cresyl violet } & Propanol & $\begin{array}{c}\text { n.r. } \\
(2.2 \mathrm{cP})^{\mathrm{a}}\end{array}$ & n.r. & n.r & 3.87 & $0.355 \pm 0.020$ & $0.696 \pm 15$ & {$[74]$} \\
\hline & 1-Propanol & 20 & 570 & 630 & $3.15 \pm 0.01$ & $0.337 \pm 0.007$ & $0.60 \pm 0.02$ & {$[56]$} \\
\hline & 1-Propanol & 25 & 578 & 660 & $3.19^{\mathrm{b}}$ & $\mathrm{Graph}^{\mathrm{c}}$ & $0.536 \pm 0.035$ & [64] \\
\hline & Water & 25 & 578 & 660 & $2.32^{\mathrm{b}}$ & n.r. & $0.117 \pm 0.010$ & {$[64]$} \\
\hline \multirow[t]{2}{*}{$\begin{array}{l}\text { Diphenyl- } \\
\text { hexatriene }\end{array}$} & $\begin{array}{l}\text { Propylene } \\
\text { glycol }\end{array}$ & 27 & 400 & 456 & $\begin{array}{c}4.53 \pm 0.03 \\
\left(\alpha_{1}=0.933\right) \\
0.91 \pm 0.11 \\
\left(\alpha_{2}=0.067\right)\end{array}$ & $0.375 \pm 0.006$ & $3.40 \pm 0.19^{d}$ & {$[75]$} \\
\hline & $\begin{array}{l}\text { Propylene } \\
\text { glycol }\end{array}$ & 27 & $800^{\mathrm{e}}$ & 456 & $\begin{array}{c}4.74 \pm 0.03 \\
\left(\alpha_{1}=0.969\right) \\
0.99 \pm 0.27 \\
\left(\alpha_{2}=0.031\right)\end{array}$ & $0.540 \pm 0.005$ & $3.58 \pm 0.10^{\mathrm{d}}$ & {$[75]$} \\
\hline \multirow[t]{2}{*}{ Rhodamine 6G } & $\begin{array}{l}\text { Propylene } \\
\text { glycol }\end{array}$ & 27 & 400 & BG39h & $\begin{array}{l}3.51 \pm 0.02 \\
(\alpha=0.984) \\
0.19 \pm 0.08 \\
(\alpha=0.016)\end{array}$ & $0.088 \pm 0.004$ & $6.16 \pm 1.10^{\mathrm{d}}$ & {$[75]$} \\
\hline & $\begin{array}{l}\text { Propylene } \\
\text { glycol }\end{array}$ & 27 & $800^{\mathrm{e}}$ & BG39h & $\begin{array}{c}3.50 \pm 0.02 \\
\alpha=0.984 \\
0.16 \pm 0.06 \\
\alpha=0.016\end{array}$ & $0.496 \pm 0.011$ & $8.19 \pm 0.69$ & {$[75]$} \\
\hline \multirow[t]{2}{*}{ WT-GFP } & $\mathrm{pH} 8^{\mathrm{g}}$ & 22 & 400 & 514 & $3.16 \pm 0.03$ & 0.36 & $16 \pm 1$ & {$[76]$} \\
\hline & $\mathrm{pH} 8^{\mathrm{g}}$ & 22 & $800^{\mathrm{e}}$ & 514 & $3.10 \pm 0.03$ & 0.51 & $17 \pm 1$ & \\
\hline \multirow[t]{3}{*}{ Perylene } & Glycerol & 25 & 256 & 448 & $\begin{array}{c}\text { n.r. } \\
(4.5-4.8)\end{array}$ & $\begin{array}{c}0.10 \\
-0.24\end{array}$ & $\begin{array}{l}28 \\
4.1\end{array}$ & $\begin{array}{l}{[66]} \\
{[67]}\end{array}$ \\
\hline & & 25 & 430 & 448 & & 0.10 & 28 & \\
\hline & & & & & & 0.24 & 4.1 & \\
\hline \multirow[t]{3}{*}{ Rhodamine 101} & Glycerol & 23 & 575 & 615 & 3.87 & $0.400 \pm 0.002$ & $120 \pm 6$ & {$[39]$} \\
\hline & $\begin{array}{l}\text { Acidified } \\
\text { ethylene } \\
\text { glycol }\end{array}$ & 23 & 575 & 615 & 4.00 & $0.400 \pm 0.004$ & 2.5 & \\
\hline & $\begin{array}{l}\text { PMMA } \\
\text { thin film }\end{array}$ & 23 & 575 & 615 & 3.8 & $0.396 \pm 0.014$ & 0 & \\
\hline
\end{tabular}

n.r.: not reported.

aViscosity.

${ }^{b}$ Uncertainty $<20$ ps.

${ }^{\mathrm{c}}$ Data represented only graphically.

${ }^{\mathrm{d}}$ Assuming a non-associative model.

eTwo-photon excitation.

${ }^{f}$ Wild-type green fluorescent protein

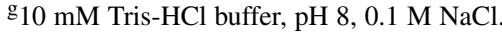

${ }^{\mathrm{h}} \mathrm{BG} 39$ filter range is $320-650 \mathrm{~nm}$. 


\section{IV.5 Identifiability analysis of time-dependent fluorescence anisotropy}

Analysis of experimental data might yield large uncertainties and high correlations between the recovered parameter values. This could erroneously be ascribed to numerical ill-conditioning. However, it has to be verified whether the model parameters can be recovered at all (i.e., the model is identifiable or not). A deterministic identifiability analysis on an error-free time-resolved fluorescence data has to be executed to verify whether the parameters of a particular model can be determined. This analysis may also provide the minimal experimental conditions under which this will become possible. Identifiability analysis can be based on the concept of a compartmental model. In a photophysical context, a compartment is a subsystem composed of a distinct type of species that acts kinetically in a unique way. Also, the motion of a Brownian rotor can be modeled in terms of a compartmental model $[44,69]$.

It has been shown that for a cylindrically symmetric rotor the diffusion coefficients $D_{\|}$and $D_{\perp}$ can be uniquely defined [70]. However, the polar and azimuthal angles defining the absorption and emission transition moments in the molecular reference frame are not individually identifiable. Nevertheless, the difference between the polar angles of these transition moments is uniquely determined.

The identifiability analysis can be extended to the case of intermolecular excited-state reactions where each of the species involved has distinct rotational diffusion characteristics. To make an identifiability analysis feasible, one assumes that all the rate constants are independent of the instantaneous orientation of the species and that there is no change in the principal axes of rotation on interconversion between the species. It is shown that, from polarized time-resolved decay data surfaces collected at two concentrations of the coreactant and three appropriately chosen emission wavelengths, (a) a unique set of rate constants for the overall excited-state process is always obtained and (b) the rotational diffusion constants and geometrical factors associated with the different anisotropy decay components can be uniquely determined and assigned to each species [44].

Evidently, the case of an intramolecular excited-state reaction is more difficult. In this case, two sets of kinetic parameters (i.e., combinations of deactivation/exchange rate constants, and rotational diffusion coefficients) exist, but these parameters cannot be assigned unequivocally to a specific species [71,72].

\section{MEMBERSHIP OF SPONSORING BODIES}

Membership of the IUPAC Physical and Biophysical Chemistry Division Committee for the period 2012-2013 is as follows:

President: K. Yamanouchi (Japan); Vice President: R. Marquardt (France); Secretary: A. Wilson (USA); Past President: A. McQuillan (New Zealand); Titular Members: K. Bartik (Belgium); A. Friedler (Israel); A. Goodwin (USA); R. Guidelli (Italy); A. Russell (UK); J. Stohner (Switzerland); Associate Members: V. Barone (Italy); A. Császár (Hungary); V. Kukushkin (Russia); V. MiškovićStanković (Serbia); Á. Mombrú Rodríguez (Uruguay); X. S. Zhao (China); National Representatives: K. Bhattacharyya (India); J. Cejka (Czech Republic); S. Hannongbua (Thailan); M. Koper (Netherlands); A. J. Mahmood (Bangladesh); O. Mamchenko (Ukraine); J. Mdoe (Tanzania); F. Quina (Brazil); N. Soon (Malaysia); V. Tomišić (Croatia).

Membership of the IUPAC Organic and Biomolecular Chemistry Division Committee for the period 2012-2013 is as follows:

President: K. Ganesh (India); Vice President: M. Garson (Australia); Secretary: A. Griesbeck (Germany); Past President: G. Koomen (Netherlands); Titular Members: G. Blackburn (UK); A. Brandi (Italy); M. Brimble (New Zealand); T. Carell (Germany); B. Han (China); F. Nicotra (Italy); Associate Members: M. Cesa (USA); V. Dimitrov (Bulgaria); H. Jacobs (Jamaica); S. H. Kang (Korea); M. Orfanopoulos (Greece); D. Sladić (Serbia); National Representatives: A. Al-Aboudi (Jordan); 
P. Crowley (Ireland); L. Dias (Brazil); E. Innocent (Tanzania); K. Khan (Pakistan); T. Krygowski (Poland); C.-C. Liao (Taiwan); D. Mizrahi (Israel); J. Streith (France); T. Vilaivan (Thailand).

Membership of the IUPAC Analytical Chemistry Division Committee for the period 2012-2013 is as follows:

President: M. Camões (Portugal); Vice President: D. Hibbert (Australia); Secretary: Z. Mester (Canada); Past President: A. Fajgelj (Austria); Titular Members: C. Balarew (Bulgaria); A. Felinger (Hungary); J. Labuda (Slovakia); M. C. Magalhães (Portugal); J. M. Pingarrón (Spain); Y. Thomassen (Norway); Associate Members: R. Apak (Turkey); P. Bode (Netherlands); Y. Chen (China); H. Kim (Korea); Y. H. Lee (Malaysia); T. Maryutina (Russia); National Representatives: A. Alam (Bangladesh); O. Chande Othman (Tanzania); L. Charles (France); M. Eberlin (Brazil); K. Grudpan (Thailand); J. Hanif (Pakistan); D. Mandler (Israel); P. el Novak (Croatia); H. Sirén (Finland); N. Torto (South Africa).

This document was prepared in the frame of IUPAC Project \#2004-021-1-300, Reference Methods, Standards and Applications of Photoluminescence. Chairs: Fred Brouwer, Enrique San Román; Members: Ulises Acuña, Marcel Ameloot, Noël Boens, Cornelia Bohne, Paul DeRose, Jörg Enderlein, Nikolaus Ernsting, Thomas Gustavsson, Niels Harrit, Johan Hofkens, Alex Knight, Helge Lemmetyinen, Hiroshi Miyasaka, Ute Resch-Genger, Alan Ryder, Trevor Smith, Mark Thompson, Bernard Valeur, Hiroyuki Yoshikawa.

\section{ACKNOWLEDGMENTS}

The authors appreciated suggestions made by Dr. Trevor Smith (University of Melbourne). Support by the Spanish Ministry of Science and Innovation 2010CTQ16457 (AUA) and the IAP P6/27 (BELSPO) Functional Supramolecular Systems (MvdV) is thankfully acknowledged.

\section{REFERENCES}

1. J. Michl, E. W. Thulstrup. Spectroscopy with Polarized Light, VCH, New York (1986).

2. D. S. Kligger, J. W. Lewis, C. E. Randall. Polarized Light in Optics and Spectroscopy, Academic Press, New York (1990).

3. (a) B. Valeur. Molecular Fluorescence. Principles and Applications, Wiley-VCH, Weinheim (2002); (b) B. Valeur, M. N. Berberan-Santos. Molecular Fluorescence. Principles and Applications, $2^{\text {nd }}$ ed., Wiley-VCH, Weinheim (2012).

4. J. R. Lakowicz. Principles of Fluorescence Spectroscopy, $3^{\text {rd }}$ ed., Springer, New York (2006).

5. C. A. Parker. Photoluminescence of Solutions, Elsevier, London (1968).

6. (a) R. F. Chen. Anal. Lett. 4, 459 (1971); (b) R. F. Chen. J. Res. Natl. Bur. Stand. A 76, 593 (1972).

7. K. Wojtuszewski, J. Knutson. In Protein Interactions: Biophysical Approaches for the Study of Complex Reversible Systems, P. Schuck (Ed.), pp. 397-416, Springer, New York (2007).

8. D. M. Jameson, G. Weber, R. D. Spencer, G. Mitchell. Rev. Sci. Instrum. 49, 510 (1978).

9. I. Gryczynski, H. Malak, J. R. Lakowicz. Chem. Phys. Lett. 245, 30 (1995).

10. D. M. Jameson, J. C. Croney, P. D. J. Moens. In Methods in Enzymology, Vol. 360, G. Marriott, I. Parker (Eds.), pp. 1-43, Elsevier (2003).

11. R. E. Dale. In Time-Resolved Fluorescence Spectroscopy in Biochemistry and Biology, R. B. Cundall, R. E. Dale (Eds.), NATO ASI Series A: Life Sciences, Vol. 69, pp. 555-612, Plenum, New York (1983).

12. J. C. Owicki. J. Biomol. Screening 5, 297 (2000).

13. T. Lindmo, H. B. Steen. Biophys. J. 18, 173 (1977).

14. C. A. Royer. Trends High Pressure Biosci. Biotechnol. 1-2, 17 (2002). 
15. J. L. Markley, D. B. Northrup, C. A. Royer (Eds.). High Pressure Effects in Molecular Biophysics and Enzymology, Oxford University Press, Oxford (1996).

16. M. E. Jolley. J. Anal. Toxicol. 5, 236 (1986).

17. S. Y. Tetin. Anal. Biochem. 307, 84 (2002).

18. S. Y. Tetin, K. M. Swift, E. D. Matayoshi. Anal. Biochem. 307, 84 (2002).

19. S. Y. Tetin, T. L. Hazlett. Methods 20, 341 (2005).

20. S. E. Braslavsky. Pure Appl. Chem. 79, 293 (2007).

21. T. Azumi, S. P. McGlynn. J. Chem. Phys. 37, 2413 (1962).

22. Ch. Buehler, C. Y. Dong, P. T. C. So, T. French, E. Gratton. Biophys. J. 79, 536 (2000).

23. F. W. J. Teale. Photochem. Photobiol. 10, 363 (1969).

24. J. Eisinger, J. Flores. Biophys. J. 48, 77 (1985).

25. D. Jameson, J. C. Croney. Comb. Chem. High Throughput Screening 6, 167 (2003).

26. H. P. Tschanz, T. H. Binkert. J. Phys. E: Sci. Instrum. 9, 1131 (1976).

27. S. R. Anderson, G. Weber. Biochemistry 8, 371 (1969).

28. A. A. Paladini, G. Weber. Rev. Sci. Instrum. 52, 419 (1981).

29. A. A. Paladini, G. Weber. Biochemistry 20, 2587 (1981).

30. P. Targowski, L. Davenport. Anal. Biochem. 274, 249 (1998).

31. J. G. Zhou, S. Koulas, P. L.-G. Chong. Rev. Sci. Instrum. 71, 4249 (2000).

32. D. Axelrod. Biophys. J. 26, 557 (1979).

33. Q. Ruan, K. Ruan, C. Balny, M. Glaser, W. W. Mantulin. Biochemistry 40, 14706 (2001).

34. M. vandeVen, M. Ameloot, B. Valeur, N. Boens. J. Fluoresc. 15, 377 (2005).

35. Commercially available from, e.g., Sigma-Aldrich, St. Louis, MO, USA.

36. O. Sire, B. Alpert, C. Royer. Biophys. J. 70, 2903 (1996).

37. R. B. Thompson, I. Gryczynski, J. Malicka. BioTechniques 32, 34 (2002).

38. L. B.-A. Johansson. J. Chem. Soc., Faraday Trans. 86, 2103 (1990).

39. T. J. V. Prazeres, A. Fedorov, S. P. Barbosa, J. M. G. Martinho, M. N. Berberan-Santos. J. Phys. Chem. A 112, 5034 (2008).

40. G. Weber, F. J. Farris. Biochemistry 18, 3075 (1979).

41. R. F. Chen, R. L. Bowman. Science 147, 729 (1965).

42. J. R. Lakowicz, I. Gryczynski, Z. Gryczynski, J. D. Dattelbaum. Anal. Biochem. 267, 397 (1999).

43. J. M. Beechem, E. Gratton, M. Ameloot, J. R. Knutson, L. Brand. In Topics in Fluorescence Spectroscopy, Vol. 2, J. R. Lakowicz (Ed.), pp. 241-305, Plenum, New York (1991).

44. J. P. Szubiakowski, R. E. Dale, N. Boens, M. Ameloot. J. Chem. Phys. 121, 7829 (2004).

45. A. Szabo. J. Chem. Phys. 81, 150 (1984).

46. W. van der Meer, H. Pottel, W. Herreman, M. Ameloot, H. Hendrickx, H. Schröder. Biophys. J. 46, 515 (1984).

47. A. Arcioni, R. Tarroni, C. Zannoni. J. Chem. Soc., Faraday Trans. 89, 2815 (1993).

48. W. E. Blumberg, R. E. Dale, J. Eisinger, D. Zuckerman. Biopolymers 13, 1589 (1974).

49. L. Brand, M. L. Johnson (Eds.). Numerical Computer Methods, Methods in Enzymology, Vol. 210, Academic, San Diego (1992).

50. Ph. Wahl. Biophys. Chem. 10, 91 (1979).

51. C. W. Gilbert. In Time-Resolved Fuorescence Spectroscopy in Biochemistry and Biology, B. Cundall, R. E. Dale (Eds.), NATO ASI Series A: Life Sciences, Vol. 69, pp. 605-606, Plenum, New York (1983).

52. J. R. Knutson, J. M. Beechem, L. Brand. Chem. Phys. Lett. 102, 501 (1983).

53. M. Ameloot, H. Hendrickx. Biophys. J. 44, 27 (1983).

54. A. Cross, G. R. Fleming. Biophys. J. 46, 45 (1984).

55. S. R. Flom, J. H. Fendler. J. Phys. Chem. 92, 5908 (1988).

56. M. Crutzen, M. Ameloot, N. Boens, R. M. Negri, F. C. De Schryver. J. Phys. Chem. 97, 8133 (1993). 
57. N. Boens, W. Qin, N. Basarić, J. Hofkens, M. Ameloot, J. Pouget, J. P. Lefèvre, B. Valeur, E. Gratton, M. vandeVen, N. D. Silva, Y. Engelborghs, K. Willaert, A. Sillen, G. Rumbles, D. Phillips, A. J. W. G. Visser, A. van Hoek, J. R. Lakowicz, H. Malak, I. Gryczynski, A. G. Szabo, D. T. Krajcarski, N. Tamai, A. Miura. Anal. Chem. 79, 2137 (2007).

58. E. Feinstein, G. Deikus, E. Rusinova, E. L. Rachofsky, J. B. A. Ross, W. R. Laws. Biophys. J. 84, 599 (2003).

59. M. N. Berberan-Santos. J. Lumin. 50, 83 (1991).

60. M. N. Berberan-Santos, P. Choppinet, A. Fedorov, L. Jullien, B. Valeur. J. Am. Chem. Soc. 122, 11876 (2000).

61. A. K. Livesey, J. C. Brochon. Biophys. J. 52, 693 (1987).

62. C. R. Mateo, P. Tauc, J. C. Brochon. Biophys. J. 65, 2248 (1993).

63. E. Gratton, D. M. Jameson, R. D. Hall. Ann. Rev. Biophys. Bioeng. 13, 105 (1984).

64. G. B. Dutt, S. Doraiswamy, N. Periasamy, B. Venkataraman. J. Chem. Phys. 93, 8498 (1990).

65. J. Beechem, M. Ameloot, L. Brand. Anal. Instrum. 14, 379 (1985).

66. M. D. Barkley, A. A. Kowalczyk, L. Brand. J. Chem. Phys. 75, 3581 (1981).

67. A. Balter, J. Szubiakowski. J. Fluoresc. 3, 247 (1993).

68. J. Szubiakowski, A. Balter, W. Novak, A. Kowalczyk, K. Wisniewski, M. Wierzbowska. Chem. Phys. 208, 283 (1996).

69. N. Boens, M. Ameloot. Int. J. Quantum Chem. 106, 300 (2006).

70. N. Boens, E. Novikov, M. Ameloot. ChemPhysChem 7, 2559 (2006).

71. N. Boens, E. Novikov, J. P. Szubiakowski, M. Ameloot. J. Phys. Chem. A 109, 11655 (2005).

72. J. P. Szubiakowski, R. E. Dale, N. Boens, M. Ameloot. Chem. Phys. Lett. 438, 113 (2007).

73. G. J. Blanchard, C. A. Cibal. J. Phys. Chem. 92, 5950 (1988).

74. G. S. Beddard, T. Doust, G. Porter. Chem. Phys. 61, 17 (1981).

75. A. Volkmer, D. Hatrick, D. J. S. Birch. Meas. Sci. Technol. 8, 1339 (1997).

76. A. Volkmer, V. Subramaniam, D. J. S. Birch, T. M. Jovin. Biophys. J. 78, 1589 (2000).

Republication or reproduction of this report or its storage and/or dissemination by electronic means is permitted without the need for formal IUPAC permission on condition that an acknowledgment, with full reference to the source, along with use of the copyright symbol $\odot$, the name IUPAC, and the year of publication, are prominently visible. Publication of a translation into another language is subject to the additional condition of prior approval from the relevant IUPAC National Adhering Organization. 